\title{
Cuestionario para la catalogación y selección de aplicaciones con tecnología Web 3.0 para Secundaria Obligatoria
}

\author{
Questionnaire for cataloging and selecting applications with Web 3.0 technology \\ for Compulsory Secondary Education \\ Elisabet Guix i Pares; eguix1@alumno.uned.es \\ Marta Ruiz-Corbella; mruiz@edu.uned.es \\ Universidad Nacional de Educación a Distancia
}

\section{Resumen}

Presentamos el diseño y validación de un instrumento para catalogación/selección de aplicaciones con tecnología Web 3.0 para la Secundaria Obligatoria. A partir de un instrumento para seleccionar software educativo elaborado por Marqués (2002), se adaptó este instrumento a criterios pedagógicos, funcionales y tecnológicos 3.0, de acuerdo con los criterios recogidos en las últimas investigaciones sobre este tema.

El instrumento se validó mediante la técnica Delphi, con un panel de expertos formado por 15 expertos en tecnología educativa y/o profesores de la Educación Secundaria Obligatoria. Se da una aceptación generalizada de la necesidad de este instrumento para la selección de aplicaciones educativas, y la inclusión en el aula de aplicaciones del entorno digital. Se prioriza la dimensión de ayuda al profesorado relacionado con la adquisición de las nuevas competencias 3.0.

Palabras clave: Web 3.0, Secundaria Obligatoria, recursos educativos digitales 3.0

\section{Abstract}

We have included the design and validation of a questionnaire for cataloging and selecting applications with Web 3.0 technology for Compulsory Secondary Education, using a tool to select educational software developed by Marqués (2002). With the updated bibliographic review, this questionnaire was adapted to educational, functional and technological criteria 3.0.

The questionnaire was evaluated using the Delphi method, and a team of 15 experts in educational technology and / or teachers of Compulsory Secondary Education. They agreed that it was necessary for the selection of educational application programs, and that the introduction of digital applications in the classroom was an urgent requirement. They stressed on the need to promote training on the new 3.0 competencies among teachers.

Keywords: Web 3.0, Compulsory Secondary Education, digital educational resources 3.0 


\section{INTRODUCCIÓN}

Nadie cuestiona la dificultad que entraña la selección de los recursos educativos más adecuados, y con criterios pedagógicos sólidos, para el proceso de aprendizaje. Elección que se complica aún más con la incorporación de las tecnologías de la información y comunicación (TIC) en las aulas, especialmente a partir de la expansión de la Web 2.0 y 3.0. Esta última es una versión ampliada de la anterior, por lo que, y tal como indican Dominic, Francis \& Pilomenraj (2014), la Web 3.0 integra todo un conjunto de nuevas tecnologías informáticas como la representación 3D, Big Data, Web Semántica, Realidad Virtual y Aumentada, Inteligencia Artificial, etc., que contribuyen a la introducción de metodologías emergentes que abren nuevas posibilidades de enseñanza/ aprendizaje al entorno educativo (Sánchez, Prendes, Martínez, Carmichael, y Martínez, 2011; Carmichael \& Tscholl, 2013).

Esta dificultad se acrecienta con el continuo lanzamiento de aplicaciones y herramientas tecnológicas en periodos cada vez más cortos de tiempo, lo que origina un exceso de recursos, que impide la eficiencia en esta selección. Además, no podemos obviar que la continua irrupción en el mercado de nuevas herramientas supera la capacidad de conocerlas, valorarlas y, en su caso, utilizarlas en el aula. Por este motivo, es necesario disponer de instrumentos que permitan seleccionar y/o valorar esos recursos digitales para el entorno web 3.0. Diferenciando el significado de recursos digitales 3.0 que no todos pueden ejecutarse en el navegador, frente a los de tecnología Web que requieren conexión a la red y navegador, lo que conlleva que se utilicen en distintos dispositivos electrónicos.

Con este propósito presentamos el proceso de elaboración y validación de un instrumento dirigido a la selección y valoración de aplicaciones web 3.0 como recurso educativo para los alumnos/as de la Educación Secundaria Obligatoria (ESO). De los instrumentos existentes para la selección de software educativo, elegimos, como punto de partida, el que elaboró Pere Marqués (2002), al responder de manera clara, útil y estructurada a las necesidades del profesorado de esta etapa. El problema al que nos enfrentamos es que este fue elaborado hace 2 décadas y, desde entonces, la tecnología ha evolucionado de tal manera que exige instrumentos que respondan a las actuales características tecnológicas.

La revisión de la literatura especializada indica que la selección, evaluación y/o catalogación del software para uso educativo se inició en la década de los ochenta del pasado siglo (Lathrop \& Goodson, 1983; Johnson, 1986; Squirres y MacDougall., 1997; Lara-Meloy, Flores, Jeffers, Llorente, Weagle, Burns, \& Davis-Kay, 2004; Ramírez, 2005; Cova, Arrieta y Riveros, 2008; Bokhove \& Drijvers, 2010; García-Barrera, 2016). A lo largo de estos años una de las dificultades recurrentes que mencionan estos autores es la fugacidad que subyace en todo diseño tecnológico, realidad que dificulta la identificación de criterios precisos y adecuados para su selección e implementación en las aulas. E identifican unos criterios comunes que deben estar presentes en todo proceso de valoración y selección, como son, p.e., adecuación, claridad, consistencia, usabilidad, navegabilidad, fiabilidad, temporalidad, actualización, utilidad, credibilidad e interactividad (García Barrera, 2016), que facilitan el poder plantear un instrumento válido y con mayor permanencia para su utilización en el aula. Analizados todos estos instrumentos nos encontramos también con diferentes perspectivas para la valoración de objetos, la calidad educativa, el tipo de recurso digital, la asignatura o el nivel educativo al que se dirige la aplicación. Coincidimos con estos autores en que el objeto a evaluar o 
seleccionar no siempre debe entenderse de la misma forma ${ }^{1}$, aunque sí afirmamos que debe haber un elemento común e indispensable en todas aquellas que estén destinadas al aprendizaje. Los primeros instrumentos se centraron en evaluar el contenido, las instrucciones, objetivos didácticos, documentación y características técnicas del material educativo digital, por lo que fueron diseñados como listas de comprobación (Cova, et al, 2008).

Sin embargo, la tendencia para evaluar y seleccionar tecnologías educativas 2.0 y 3.0 se enfocaron hacia los procesos colaborativos (Kelly, 2008), con el objetivo de promover la discusión, incluyendo a los actores implicados en el proceso evaluativo, a la vez que impulsar la utilización de estas tecnologías en contextos reales de aprendizaje. Hadjerrouit (2010) aporta un interesante estudio sobre los recursos basados en tecnologías web (Web based learning resources - WBLR), en el que propone un marco conceptual para explorar su diseño y evaluación en el contexto escolar. Delimita los WBLR como un objeto de aprendizaje que existe en la intersección de contenido, pedagogía y tecnología. A partir de estos criterios establece los principales indicadores asociados a cada uno de estos, p.e., contenido de fácil acceso y lectura, páginas bien estructuradas; navegación intuitiva y sencilla, etc.

En lo que concierne a recientes investigaciones relacionadas con la búsqueda de criterios para la selección y evaluación de recursos digitales, en el panorama internacional, destacamos la de Paganelli (2016) y la de Karolcík, Cipková, Veselský, Hrubišková, \& Matulcíková (2017). Ambas se centran en la premisa de que la selección cuidada de material de alta calidad asociado a tecnologías de apoyo puede ayudar a mejorar la participación de los estudiantes.

A partir de esta revisión bibliográfica se reelaboró un nuevo instrumento dirigido a la evaluación y catalogación de las aplicaciones educativas diseñadas para todos los cursos de Secundaria Obligatoria (12 - 16 años) en un entorno web 3.0. El punto de partida fue el instrumento elaborado por Marqués (2002), actualizando los aspectos tecnológicos en el apartado de catalogación, además de aquellos más funcionales adaptados a las necesidades de esta etapa educativa. También se reelaboró el bloque de selección/evaluación de software adaptándolo a las aplicaciones de la web 3.0 y sus efectos pedagógicos y didácticos en el aula. En suma, se trata de un instrumento estructurado en nueve apartados:

1. Glosario web 3.0

2. Identificación de la aplicación

3. Objetivos educativos

4. Categorización de la aplicación

5. Aspectos tecnológicos necesarios para el uso de la aplicación (dispositivos físicos, aplicaciones, sistema de ayuda, seguridad, etc.)

6. Aspectos pedagógicos asociados al uso educativo, que se categorizan atendiendo al tipo de aprendizaje, apoyo al profesorado en el proceso educativo y debilidades / barreras a superar

\footnotetext{
${ }^{1}$ En este punto es interesante referirnos al debate existente entre los autores que prefieren hablar de 'objetos de aprendizaje' o 'Learning Objects' (García-Aretio, 2005; Kay \& Knaack, 2007; Hadjerrouit, 2010), los que apelan a los recursos educativos o aquellos que han trabajado con el software educativo (Marquès, 2002; Bayram \& Nous, 2004). Ahora, todos ellos son recursos digitales, algunos de ellos reutilizables, que funcionan en distintos sistemas operativos (Windows, Linux, Apple, etcétera) y presentan una clara dimensión educativa.
} 
7. Competencias educativas que desarrollan

8. Observaciones a tener en cuenta en esa aplicación

9. Valoración global cualitativa

Del instrumento originario se suprimió lo correspondiente al mapa de navegación y descripción de actividades, al facilitar los portales actuales esta información, a la vez que favorecen la accesibilidad. El bloque específico sobre dimensiones técnicas y estéticas, se eliminó ya que se incorporó en otros apartados de la ficha. Se suprimió el bloque de esfuerzo cognitivo asociado, al estar contemplado entre las competencias clave a lograr. Y se modificó la parte correspondiente al título del recurso, incluyendo los componentes técnicos más relevantes y necesarios. En vez de temática se prefirió incluir objetivo a lograr; y el apartado sobre recursos didácticos se denominó estrategias didácticas. Este rediseño concluyó en un cuestionario inicial, que puede consultarse en https://bit.ly/2C14YH8

\section{MÉTODO}

Aunque partimos de un instrumento validado, reconocido y utilizado desde 2002, la extraordinaria evolución tecnológica exigía que se incorporaran las necesarias actualizaciones, lo que derivó en una nueva validación (Álvarez, Gisbert y González, 2013). Para ello se establecieron 2 fases, una primera en la que 2 expertos evaluaron el contenido y claridad del instrumento actualizado. Y una segunda, empleando el técnica Delphi, en la que los expertos participantes valoraron su pertinencia y claridad tras el uso en el aula de aplicaciones educativas 3.0 (López-Gómez, 2018).

En la primera fase se contó con 2 expertos, Pere Marquès, conocido especialista en tecnología de la educación y autor del instrumento referente de esta investigación. Y Jaume Ferrer, experto en Informática y Comunicaciones, con una trayectoria de más de 30 años como docente en la ESO. A ambos se les pidió su opinión sobre la pertinencia, claridad y adecuación del contenido del instrumento modificado teniendo en cuenta el contexto educativo de la ESO. Ambos coincidieron en la necesidad de incorporar al aula las novedades derivadas de la web 3.0, y propusieron como mejoras al instrumento:

- Incorporar un glosario con los nuevos términos.

- Diferenciar entre los recursos de las tecnologías Web 2.0 de los de la 3.0.

- Separar los aspectos objetivos de calidad y los contextuales del uso de la aplicación.

- Simplificar los ítems con la finalidad de no repetir conceptos, a la vez que distinguirlos claramente entre sí.

- Incorporar sistemas de ayuda, destacando el manual integrado en la propia aplicación en lenguaje hipertexto.

El instrumento resultante se dividió en 3 grandes apartados. En el primero se abordan los aspectos generales del recurso (identificación, objetivos y características técnicas de la aplicación) centrada en aspectos tecnológicos y catalogación. En el segundo se centra ya en la evaluación de los aspectos ergonómicos, funcionales y pedagógicos. Una última parte atiende las posibilidades didácticas que se derivan de la aplicación.

Una vez reelaborado este instrumento a partir de las observaciones expresadas por ambos 
expertos, se procedió a la segunda fase en la que se le sometería, a través de la técnica Delphi, a la valoración por parte de un panel de 15 profesores con amplia experiencia docente en Secundaria y/o en TIC. Lo relevante es que estos participantes valorarían los diferentes apartados del cuestionario tras el manejo y utilización en el aula de las aplicaciones seleccionadas específicamente para esta investigación.

Tal como se mencionó, y como debían ser utilizadas aplicaciones 3.0 en contextos reales, se seleccionaron aquellas que mejor se adecuaran al ámbito educativo: web semántica (dispone de elementos integrados con técnicas de Inteligencia artificial en buscadores y visualizadores web), realidad virtual y aumentada, gamificación, open data y programas que utilizan bots (Dominic et al., 2014). Otro criterio utilizado fue su accesibilidad en cuanto tres principios relevantes para su elección: formato claro y fácil seguimiento, acceso vía multi dispositivo y de acceso libre, lo que redujo el listado considerablemente. Por último, el criterio 'categoría' se recogió de la adaptación de Miranda, Isaias, \& Costa (2015). A partir de estos criterios se identificaron, finalmente, 10 aplicaciones (Tabla 1).

\begin{tabular}{ccc}
\hline Aplicación seleccionada & Categoría & Fecha edición \\
\hline Kajoot! & Gamificación & 2013 \\
Toovari & Gamificación & 2014 \\
Socrative & Gamificación & 2010 \\
Wolfram Alpha & Buscador inteligente o semántico & 2009 \\
Gapminder & Open Data & 2007 \\
Google Expeditions & Realidad Virtual & 2015 \\
Street View & Realidad Virtual & 2007 \\
DiscoveryVR & Realidad Virtual & 2015 \\
Canal de YouTube 360 & Realidad Virtual & 2015 \\
Zspace for Education & Realidad Virtual & 2007 \\
\hline
\end{tabular}

Tabla1. Aplicaciones web 3.0 seleccionadas

El siguiente paso para la validación de este instrumento (López-Gómez, 2018) se organizó en tres fases: preliminar, exploratoria y final.

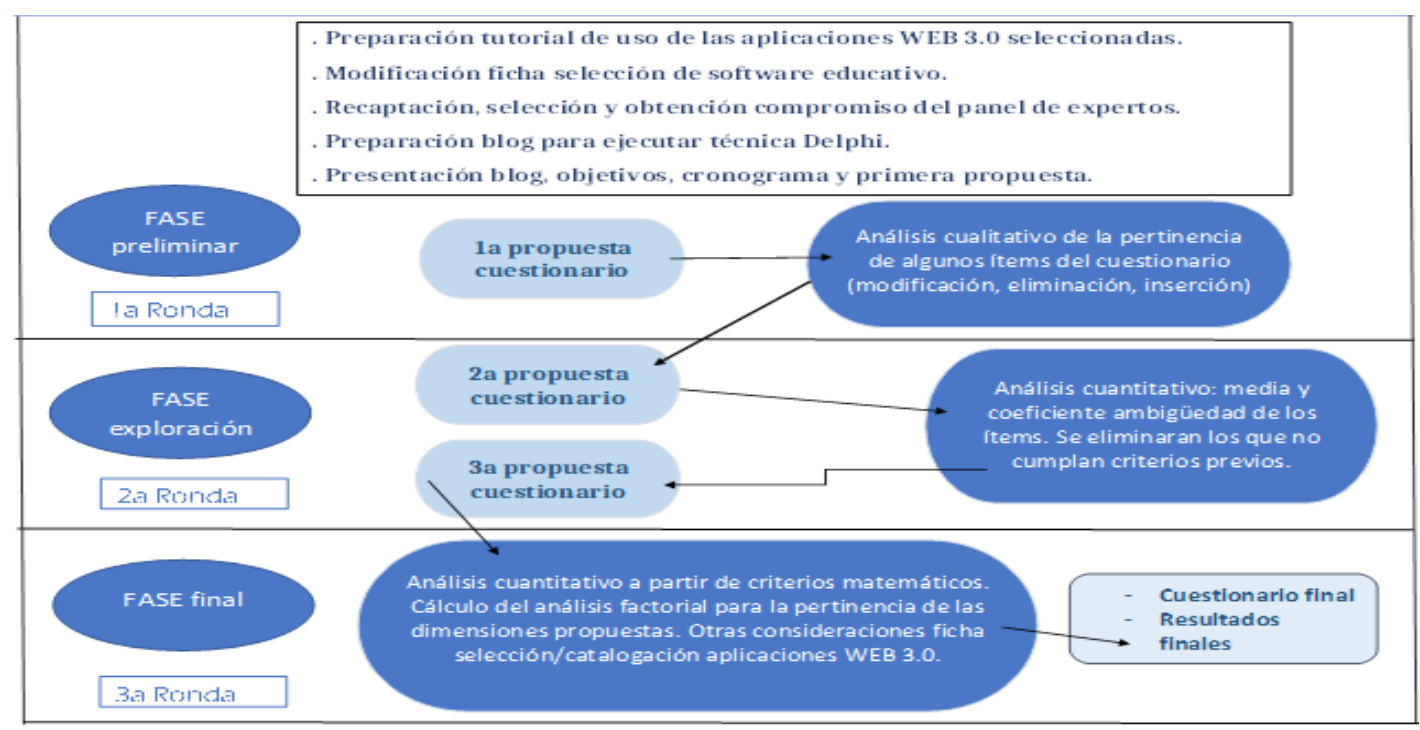

Figura 1.- Proceso de validación del instrumento para la selección de aplicaciones 3.0. 
Fase preliminar, estructurada en 4 pasos:

A) Creación de tutoriales de las aplicaciones 3.0 seleccionadas para su utilización en el aula y valoración por parte de los expertos.

B) Identificación y selección de los expertos para el panel, de acuerdo con el perfil para esta investigación: experto en TIC y/o experiencia docente en el nivel de la ESO. La selección de la muestra fue por muestreo no probabilístico por conveniencia, por la que se identificó un grupo de expertos atendiendo a la competencia de dichos candidatos en el objeto de esta investigación, valorada a partir de sus investigaciones, experiencia docente, proyectos innovadores y publicaciones difundidas en sus páginas web. Se registró de esta forma a 25 profesores (tecnólogos educativos y/o vinculados a la ESO), que fueron invitados, por correo electrónico, a formar parte de esta investigación. De estos, únicamente 15 confirmaron su participación, constituyendo un panel de expertos con una media de 22 años de experiencia profesional en Secundaria y/o tecnologías educativas. Todos recibieron por escrito el objetivo y contenido de esta investigación y su cometido se centró en valorar, tras la aplicación en el aula de las aplicaciones 3.0 seleccionadas, el bloque centrado en la dimensión pedagógica, al ser el único que se modificó de forma significativa. El contenido de dicho bloque se organizó en 29 ítems. 8 de los cuales se refieren a la detección de actitudes del alumnado ante el uso de estas aplicaciones 3.0, 7 relativos al tipo de aprendizaje que impulsan, 6 vinculados con la ayuda que pueden proporcionar al profesorado en el seguimiento del aprendizaje del alumnado y 8 referidos a uso educativo.

C) Elaboración de un blog con los contenidos de esta investigación (objetivos, contacto, fases, cronograma, tutoriales de las aplicaciones seleccionadas y resultados) para el seguimiento de los expertos (https://proyecto-web-3-0.webnode.es/)

D) Realización de la primera ronda de la técnica Delphi, tras la utilización de las aplicaciones en el aula. Valoración de aspectos pedagógicos y funcionales del instrumento, argumentando la pertinencia y adecuación de los ítems propuestos. Las respuestas se recogieron en formularios elaborados ad hoc en Google Drive y se analizaron de acuerdo a criterios de prioridad pedagógica.

Fase exploración, se desarrolló la segunda ronda centrada en una nueva revisión del bloque pedagógico del instrumento a partir de un cuestionario para expresar el grado de acuerdo con una escala Likert de 5 niveles. La decisión para la modificación o supresión de un ítem se concretó a partir de los valores de los coeficientes de ambigüedad, promedio y de la desviación típica, además de los comentarios de los expertos. En concreto, se descartaron los ítems que obtuvieron un coeficiente de ambigüedad superior o cercana a 2, o una desviación típica intensa. Con estos criterios de convergencia se ha elaborado la tercera propuesta. En esta última versión se convino consultar la información relacionada con la importancia y gestión de este tipo de instrumento, además de la confirmación de adecuación de los ítems formulados.

Fase final, en la que se empleó el mismo análisis cuantitativo y se recogió la información 
adicional referente a la importancia de uso de este instrumento en la ESO. Con los ítems finales se procedió a analizar la conveniencia de las dimensiones planteadas del cuestionario mediante análisis factorial. Por último, se presentó el informe y cuestionario final al panel de expertos a través del blog.

\section{DISCUSIÓN DE RESULTADOS}

Tras la primera ronda de la técnica Delphi, centrada en la definición y precisión de los ítems, adecuación o no incorporación de aquellos elementos no contemplados, de los 29 ítems iniciales del bloque pedagógico, se concluyó la reelaboración de los ítems 4, 5, 8 y 23 para una mejor comprensión. A la vez, se eliminaron el 1, 11, 19 y 22, al coincidir los expertos en su falta de pertinencia o formulación ambigua. No se propuso la incorporación de ningún ítem nuevo, por lo que se procedió a trabajar con un instrumento de 25 ítems.

En la segunda y tercera ronda, el bloque pedagógico del instrumento obtuvo resultados similares en los estadísticos calculados (tabla 2). En cuanto a la media, los resultados se concentran entre el 3,23 el más bajo y el 4,46 el más alto, lo que implica un alto grado de acuerdo con los ítems propuestos, aunque con alta dispersión de respuestas.

En relación al coeficiente de ambigüedad, como sabemos, aquellos superiores a 2 deben descartarse al haber mayor acuerdo entre los jueces. En consecuencia, el ítem 3 y 9 se eliminaron. Además, el ítem 11 fue suprimido al tener una elevada desviación típica, decisión también avalada por la valoración de un experto que lo consideró ambiguo en la primera fase.

\begin{tabular}{llll}
\hline \multicolumn{1}{c}{ Actitudes del alumnado } & $\mathrm{M}$ & $\mathrm{D}$ & \multicolumn{1}{c}{ CA } \\
\hline 1. Potencia la motivación. & 4,06 & 1,16 & 1,54 \\
2. Facilita la autoevaluación. & 3,40 & 1,18 & 1,66 \\
3. Ofrece más posibilidades de concentración en distintas & 3,26 & 1,28 & 2,12 \\
actividades. & & & \\
4. Ayuda al alumno a llevar a cabo un proceso reflexión de trabajo. & 3,23 & 0,72 & 1,00 \\
5. Fomenta la iniciativa. & 3,6 & 1,24 & 1,81 \\
6. Facilita el auto aprendizaje. & 4,46 & 0,64 & 1,07 \\
7. Mejora la adaptación del alumnado en distintas actividades de & 3,6 & 1,18 & 1,51 \\
aprendizaje. & & & \\
Tipo de aprendizaje. & & & \\
8. Facilita el aprendizaje cooperativo/colectivo. & 4,13 & 0,64 & 0,86 \\
9. Facilita el aprendizaje significativo. & 3,46 & 1,24 & 1,91 \\
10. Facilita el aprendizaje por experiencia. & 4,06 & 0,70 & 1,14 \\
11. Facilita el aprendizaje por descubrimiento. & 3,53 & 1,35 & 1,81 \\
12. Facilita el aprendizaje constructivista. & 3,73 & 0,79 & 1,31 \\
13. Facilita un aprendizaje que amplía el tradicional. & 4,26 & 0,70 & 1,12 \\
Ayuda al profesorado. & & & \\
14. Facilita el seguimiento en relación al ritmo de trabajo del & 3,73 & 1,09 & 1,68 \\
alumnado. & & & \\
15. Mejora en el control de los puntos débiles del alumnado. & 3,53 & 0,74 & 1,14 \\
16. Mejora en el control de los puntos fuertes del alumnado. & 3,6 & 0,82 & 1,33 \\
17. Facilita el trabajo con alumnado agrupado en distintos niveles. & 4 & 0,37 & 0,57 \\
\hline
\end{tabular}




\begin{tabular}{|c|c|c|c|}
\hline $\begin{array}{l}\text { 18. Potencia realizar actividades que antes no se hacían por } \\
\text { aspectos logísticos. }\end{array}$ & 4,13 & 0,83 & 1,51 \\
\hline \multicolumn{4}{|l|}{ Debilidades por superar } \\
\hline $\begin{array}{l}\text { 19. Facilitar diferentes actitudes/comportamientos en entornos } \\
\text { reales y virtuales }\end{array}$ & 3,33 & 1,11 & 1,81 \\
\hline 20. Potenciar el uso de NORMAS para una correcta administración. & 3 & 1,19 & 1,20 \\
\hline $\begin{array}{l}\text { 21. Fomentar espacios para la crítica y la reflexión de los contenidos } \\
\text { abordados. }\end{array}$ & 3,86 & 0,74 & 1,14 \\
\hline $\begin{array}{l}\text { 22. Facilitar información relativa a efectos perjudiciales de salud con } \\
\text { el uso de algunas tecnologías. }\end{array}$ & 3,8 & 0,86 & 1,20 \\
\hline 23. Fomentar la verificación de los resultados obtenidos. & 3,6 & 1,05 & 1,77 \\
\hline 24. Facilitar el uso responsable de las aplicaciones con los demás. & 3,93 & 0,96 & 1,56 \\
\hline 25. Potenciar implicación parental para seguimiento. & 4,13 & 0,74 & 1,14 \\
\hline
\end{tabular}

Tabla 2. Resultado estadísticos 2a ronda del DELPHI

En la tercera ronda, tras el estudio de sus resultados, los únicos ítems que recogieron resultados distintos fueron el 5, 7 y 19, que también fueron suprimidos (tabla 3). Lo que concluyó en un instrumento compuesto por 19 ítems avalado por un moderado nivel de pertinencia, junto con un satisfactorio grado de acuerdo entre los expertos.

\begin{tabular}{|c|c|c|c|}
\hline Actitudes del alumnado & & & \\
\hline 5. Fomenta la iniciativa. & 3,4 & 1,24 & 1,93 \\
\hline $\begin{array}{l}\text { 7. Mejora la adaptación del alumnado en distintas actividades de } \\
\text { aprendizaje. }\end{array}$ & 3,4 & 1,29 & 1,90 \\
\hline \multicolumn{4}{|l|}{ Debilidades por superar } \\
\hline $\begin{array}{l}\text { 19. Facilitar diferentes actitudes/comportamientos en entornos } \\
\text { reales y virtuales }\end{array}$ & 3,26 & 1,03 & 1,62 \\
\hline
\end{tabular}

Tabla 3. Resultados discrepantes en el análisis cuantitativo 3ạ ronda del Delphi.

A partir de estas valoraciones se elaboró ya el instrumento definitivo, al que se denominó Cuestionario para la catalogación y selección de aplicaciones con tecnología WEB 3.0 para la ESO (Anexo). Se realizó el análisis factorial con el objetivo de valorar la idoneidad y conveniencia tanto del número de ítems definitivo, como de las dimensiones o factores que lo explican.

Al concluir la tercera ronda, previo al análisis factorial, se acudió, además, al análisis de correlación bivariado entre todas las variables (ítems) implicadas, con objeto de observar las posibles relaciones elevadas que pudieran producirse entre distintas parejas de ítems, que, a nivel estadístico resultarían aquellas que superan o se acercan al valor de 0,75. Y se propuso la eliminación de aquellas relaciones elevadas. En concreto, se consensuó la supresión del ítem 15 , ya que la revisión teórica apuntó a la de contenido entre la pareja de ítems 15 y 16 $(r=0,720) y$, por otro, con 15-24 ( $r=0,753)$, por lo que el cuestionario pasó a contar 18 ítems. Continuando con la revisión de los restantes, se detectó en los ítems 21 y 23 una relación elevada $(0,75)$, a la vez que el ítem 21 comprehende al 23, motivo por el cual se eliminó.

Calculando el análisis factorial (SPSS versión 24) de 17 ítems, se recuperó la idea inicial de organizarlo en 4 dimensiones que sustentarían la capacidad explicativa de los ítems 
resultantes. Se reflexionó sobre la idoneidad de estos factores y se planteó la idea del instrumento basado en tres dimensiones, ya que se detectó categorías con un solo ítem. Se recuperó la información cuantitativa con las correlaciones elevadas entre los pares de ítems: 2 y $14(r=0,747)$, por lo que se eliminó el ítem 14, al ser redundante y fácilmente ubicable en otras dimensiones. Se repitió para los ítems 20 y 22 ( $r=0,624)$, fácilmente rastreables en otras dimensiones, eliminando el ítem 20, con el menor promedio de todos. Y finalmente, los ítems 1 y 2 , se eliminaron, al mostrar un comportamiento similar a los anteriores.

Con objeto de continuar ofreciendo un sustrato estadístico a estos resultados y apoyados en las reflexiones derivadas del análisis factorial, unido al hecho de que se cuenta con un escaso tamaño muestral de 15 expertos, se forzó la presentación final de ítems del instrumento a la mínima expresión susceptible de ser validada por este. En esta última revisión, se obtuvo la mínima idoneidad, por lo que se debe proceder con cautela con estos resultados, puesto que la prueba KMO no obtuvo la suficiente idoneidad que se debería esperar (0,163 en este caso).

En lo concerniente a la presentación de dimensiones adecuadas, el análisis factorial inicial propone un modelo de varianzas explicadas que optaría por cinco componentes. Tras su revisión, se observó que las dimensiones 3,4 y 5 tan sólo son sustentadas por un único ítem; ante tal circunstancia forzamos el modelo hacia 4 componentes, con el cual también volvemos obtener para el cuarto una saturación por medio de 1 solo ítem. Finalmente se llegó al análisis de tres componentes, en el cual se detectó una presentación de saturación oportuna.

Además, existe una aceptación generalizada de los ítems propuestos en cada una de las dimensiones planteadas, destacando los correspondientes a las dimensiones de ayuda al profesorado y debilidades a superar en el uso de aplicaciones con tecnología Web 3.0. Esto refuerza las premisas del marco teórico referente al uso educativo de las tecnologías Web 3.0 (Sánchez et al., 2011; Carmichael \& Tscholl, 2013).

El cálculo del estadístico Alpha de Cronbach obtiene un 0,730, lo que nos indica que el análisis de fiabilidad del cuestionario completo es aceptable (De Vet, Mokkink, Mosmuller \& Terwee, 2017). Ahora, no se debe perder de vista que estos resultados son solo significativos para la muestra de este estudio, aunque revela un valor como estudio piloto, que debe ser replicado con un mayor número de profesionales, lo que mejoraría las propiedades del bloque. En suma, se logra aportar una herramienta final simplificada, basada en un modelo de tres factores y con un total de 13 ítems, que explican el 60\% de la varianza final de los datos y con una gran consistencia interna, capaz de ofrecer mayor calidad en cada uno de sus apartados.

Por último, cabe destacar que la mayoría de los expertos se pronunciaron a favor de la necesidad de utilizar esta herramienta proyectada en el contexto educativo de la ESO, ya que no conocen ninguna otra para este fin. Estos datos están en concordancia con la escasa bibliografía que existe sobre tecnologías emergentes relacionadas con el nivel de Secundaria. A la vez, todos coinciden en la necesidad de promover en los centros educativos la figura del experto TIC, que impulsen la inclusión de estas aplicaciones 3.0 en las aulas de la ESO. 


\section{CONCLUSIONES}

En definitiva, la validación de este instrumento aporta una primera referencia como instrumento dirigido a la evaluación y catalogación de los recursos educativos digitales de la web 3.0 en el nivel de la ESO. Además de sensibilizar al profesorado para la inclusión en el aula de estos recursos educativos propios del entorno digital en el que están creciendo nuestras alumnas y alumnos, fomentando, de este modo, la formación en competencias digitales 3.0: información y alfabetización informacional, comunicación y colaboración, creación de contenidos digitales, seguridad y resolución de problemas (INTEF, 2017).

En cuanto al instrumento, a nivel teórico, y en concordia con el panel de expertos consultados, existe una aceptación generalizada de los ítems propuestos en las dimensiones planteadas. Destacan con más intensidad los ítems correspondientes a las dimensiones de ayuda al profesorado y debilidades a superar en el uso de aplicaciones con tecnología Web 3.0. Destaca el ítem asociado a "fomenta el auto aprendizaje" de la categoría de mejoras para el aprendizaje del alumnado, al obtener la media más elevada. Por otra parte, el ítem "potenciar el uso de normas para una correcta administración", fue el de promedio más bajo.

Es interesante destacar el punto correspondiente a qué sujetos deben ser los encargados de gestionar y difundir el instrumento propuesto, en el que coinciden en la importancia de la figura del experto TIC en los centros educativos, no coincidente con el profesorado. Se ha añadido el apartado nivel de dificultad digital a la ficha por alusión de más de 4 expertos y considerarse de relevancia.

También cabe destacar que la gran mayoría de estos profesionales no estaban familiarizados con las aplicaciones basadas en inteligencia artificial (Open data y buscadores semánticos), lo que nos indica la novedad de este tipo de recursos y la necesaria inclusión de la dimensión de alfabetización informacional. Esto coincide con Cervera y Álvarez (2015), que ya plantearon la necesidad de un plan formativo de esta competencia para el profesorado de la ESO.

\section{REFERENCIAS BIBLIOGRÁFICAS}

Álvarez, J.F., Gisbert, M., y González, J. (2013). Validación de un cuestionario para medir el nivel de alfabetización informacional digital del profesorado de educación secundaria de todo el Estado español. XVI Congreso Internacional EDUTEC 2013 [Actas]. San José, Costa Rica, 6-7 noviembre. Recuperado de $\underline{\text { https://bit.ly/2LxPsbn }}$

Bayram, S. \& Nous, A.P. (2004). Evolution of educational software evaluation: Instructional software assessment. Turkish Online Journal of Educational Technology, 3(2), 21 - 27.

Bokhove, C., \& Drijvers, P. (2010). Digital tools for algebra education: Criteria and evaluation. International Journal of Computers for Mathematical Learning, 15(1), . 45-62.

Carmichael, P. \& Tscholl, M.J. (2013). Cases, Simulacra, and Semantic Web

Technologies. Journal of Computer Assisted Learning, 29, 31-42. 
Cervera, M.G., y Álvarez, J.F. (2015). Grado de alfabetización informacional del

profesorado de Secundaria en España: creencias y autopercepciones. Comunicar, 45, 187194. doi:10.3916/C45-2015-20

Cova, A., Arrieta, X., y Riveros, V. (2008). Análisis y comparación de diversos modelos de evaluación de software educativo. Enl@ce: Revista Venezolana de Información, Tecnología y Conocimiento, 5(3), 45-67.

De Vet, H.C.B, Mokkink, L.B., Mosmuller, D.G., \& Terwee, C.B. (2017). Spearman-

Brown prophecy formula and Cronbach's Alpha: Different faces of reliability and opportunities for new applications. Journal of Clinical Epidemiology, 85, 45 - 49. doi:10.1016/j.jclinepi.2017.01.013

Dominic, M., Francis, S., \& Pilomenraj, A. (2014). E-learning in web 3.0. International Journal of Modern Education and Computer Science, 6(2), 8-14. doi:10.5815/ijmecs.2014.02.02

García Aretio, L. (2005). Objetos de aprendizaje. Características y repositorios.

Boletín Electrónico de Noticias de Educación a Distancia (BENED), abril. Recuperado de https://bit.ly/2OIWyOD

García-Barrera, A. (2016). Evaluación de recursos tecnológicos didácticos mediante e-rúbricas. RED. Revista de Educación a Distancia, 49, 1-13.

Hadjerrouit, S. (2010). A conceptual framework for using and evaluating web-based learning resources in school education. Journal of Information Technology Education, 9, 53-79.

Instituto Nacional de Tecnologías Educativas y de Formación del Profesorado (2017). Marco Común de Competencia Digital Docente. Madrid: Ministerio de Educación, Cultura y Deporte / INTEF. Recuperado de https://bit.ly/2jakssz

Johnson, W.E. (1986). Evaluation of computer software for use in the classroom [Paper]. National Conference of the National Council of States on Inservice Education, Nashville, EEUU.

Kay, R.H. \& Knaack, L. (2007). Evaluating the learning in learning objects. Open Learning, 22(1), 5-28.

Karolcík, Š., Cipková, E., Veselský, M., Hrubišková, H., \& Matulcíková, M. (2017). Quality

parameterization of educational resources from the perspective of a teacher. British Journal of Educational Technology, 48(2), doi:http://dx.doi.org.ezproxy.uned.es/10.1111/bjet.12358

Kelly, G. (2008). A collaborative process for evaluating new educational technologies. Campus-Wide Information Systems, 25(2), 105-113.

Lara-Meloy, T., Flores, E., Jeffers, L., Llorente, C., Weagle, V., Burns, M., ～\& Davis-Kay, J. 
(2004). Selecting educational software for teenagers in after- school settings: A toolkit from the America connects consortium. Newton, MA, EEUU: Education Development Center.

Lathrop, A. \& Goodson, B. (1983). Courseware in the classroom: Selecting, organizing, and using educational software. Reading, MA, EEUU: Addison-Wesley Publishing Co.

López Gómez, E. (2018). El método Delphi en la investigación actual: una revisión teórica y metodológica. Educación XX1, 21(1), 17-40. doi: 10.5944/educXX1.15536

Marquès, P. (2002). Evaluación y selección de software educativo. Comunicación y Pedagogía, $185,31-37$.

Miranda, P., Isaias, P., \& Costa, C.J. (2015). E-Learning and Web Generations: Towards

Web 3.0 and E-Learning 3.0. International Proceedings of Economics Development and Research, 81, 92-103.

Paganelli, A. (2016). Storytime in a digital world: Making a case for thinking outside the

book. Knowledge Quest, 44(3), 8-17.

Ramírez, E. (2005). Selección de software educativo: Dos ejemplos de evaluación de material didáctico en soporte CD-Rom. Cultura y Educación, 17(2), 131-145. doi:10.1174/1135640054192838

Sánchez, M.M.; Prendes, M.P.; Martínez, F.; Carmichael, P., y Martínez, A. (2011).

Experiencias de incorporación de aplicaciones semánticas a la educación. EDUTEC, Revista Electrónica de Tecnología Educativa, 36, 1-18.

Squires, D., y MacDougall, A. (1997). Cómo elegir y utilizar software educativo: Guía para el profesorado. Madrid: Morata.

\section{Para referenciar este artículo:}

Guix i Pares, E., \& Ruiz-Corbella, M. (2019). Cuestionario para la catalogación y selección de aplicaciones con tecnología Web 3.0 para Secundaria Obligatoria. Edutec. Revista Electrónica De Tecnología Educativa, (66), 16. https://doi.org/10.21556/edutec.2018.66.1171 


\section{Anexo}

Ficha final

Ficha para catalogar y evaluar aplicaciones con tecnología Web 3.0 para la Educación Secundaria Obligatoria -ESO

Adaptado de Marquès, 2002

APP = Aplicación informática, se ejecutan en la Web o en un dispositivo móvil, instalado directamente en local.

WEB 2.0 = Evolución de la gran red Internet, que ofrece a los usuarios la capacidad de interactuar y colaborar entre sí, como creadores de contenido, en una comunidad. Algunos ejemplos son las redes sociales, wikis y blogs.

WEB 3.0 = Versión actualizada de la Web 2.0. Ofrece aplicaciones que permiten funcionalidades nuevas o distintas a las de la tecnología 2.0., por ejemplo: contenidos semánticos, búsquedas de lenguaje natural, tecnologías de inteligencia artificial, etc.

REA = Recursos educativos abiertos. Materiales de aprendizaje en soporte digital de carácter gratuito, publicados con una licencia abierta que permite su uso, adaptación y redistribución por otros sin ninguna restricción o limitación.

RA = Realidad aumentada. Conjunto de dispositivos que añaden información virtual a la información física ya existente.

$\mathrm{RV}=$ Realidad virtual. Entorno u objetos de apariencia real, que simulan la realidad (se usan gafas u otros elementos para su uso).

OPEN DATA = Conjuntos de datos a disposición del público, que pueden ser reutilizados sin ninguna restricción.

BUSCADOR SEMÁNTICO = Buscadores evolucionados e "inteligentes" que permiten obtener resultados más eficientes.

OTROS:

IDENTIFICACIÓN APLICACIÓN

Título:

Plataforma: $\quad$ App móvil $\square$ App WEB $\square$ Otros

URL de acceso: Https://

Acceso al material: REA $\square$ De prueba $\square$ Pago o licencia privativa $\square$ Otros

Autores / Licencia: Haga clic o pulse aquí para escribir texto. 


\begin{abstract}
Versión: $\quad$ Haga clic o pulse aquí para escribir texto.
Contacto: $\quad$ Haga clic o pulse aquí para escribir texto.

Fecha de creación: Haga clic o pulse aquí para escribir texto.

Conocimiento digital para su uso: Escaso $\square$ Medio $\square$ Alto $\square$

Otras consideraciones:
\end{abstract}

\title{
OBJETIVOS EDUCATIVOS A LOGRAR
}

Categorización del aplicativo. (Seleccionar la opción de la lista correspondiente)

Elija un elemento.

Aspectos tecnológicos. (Marcar con una X en cada elemento que se identifique)

$$
\text { DISPOSITIOS FÍSICOS NECESARIOS }
$$

Dispositivos móviles $\square \quad$ Impresora $\square \quad$ Teclado especial $\square \quad$ Micrófonos $\square$
DVD $\quad \square$ USB $\square \quad$ Pantallas especiales $\square \quad$ Kits especiales $\square \quad$ Gafas $\square$

Auriculares $\square \quad$ Otros Haga clic o pulse aquí para escribir texto.

APLICACIONES Y/O PROGRAMAS NECESARIOS

Haga clic o pulse aquí para escribir texto

\section{SISTEMA DE AYUDA O ORIENTACIÓN}

Manual digital (por ejemplo, un PDF) $\square \quad$ Manual integrado en la aplicación $\square$

Video tutorial $\square$ Correo electrónico $\square$

Atención telefónica $\square \quad$ Chat $\square \quad$ Foros $\square$

Otros Haga clic o pulse aquí para escribir texto.

\section{AMENAZAS POSIBLES DE SEGURIDAD}

Confidencialidad $\square \quad$ Suplantación de identidad $\square$

Ataques externos $\square \quad$ Otros Haga clic o pulse aquí para escribir texto. 
Aspectos pedagógicos relacionados con el uso como producto de estas herramientas en el aula.

Muy Baja Normal Alta Muy

(Marcar con una $X$ en el nivel adecuado a la baja alta afirmación presentada en la casilla que proceda)

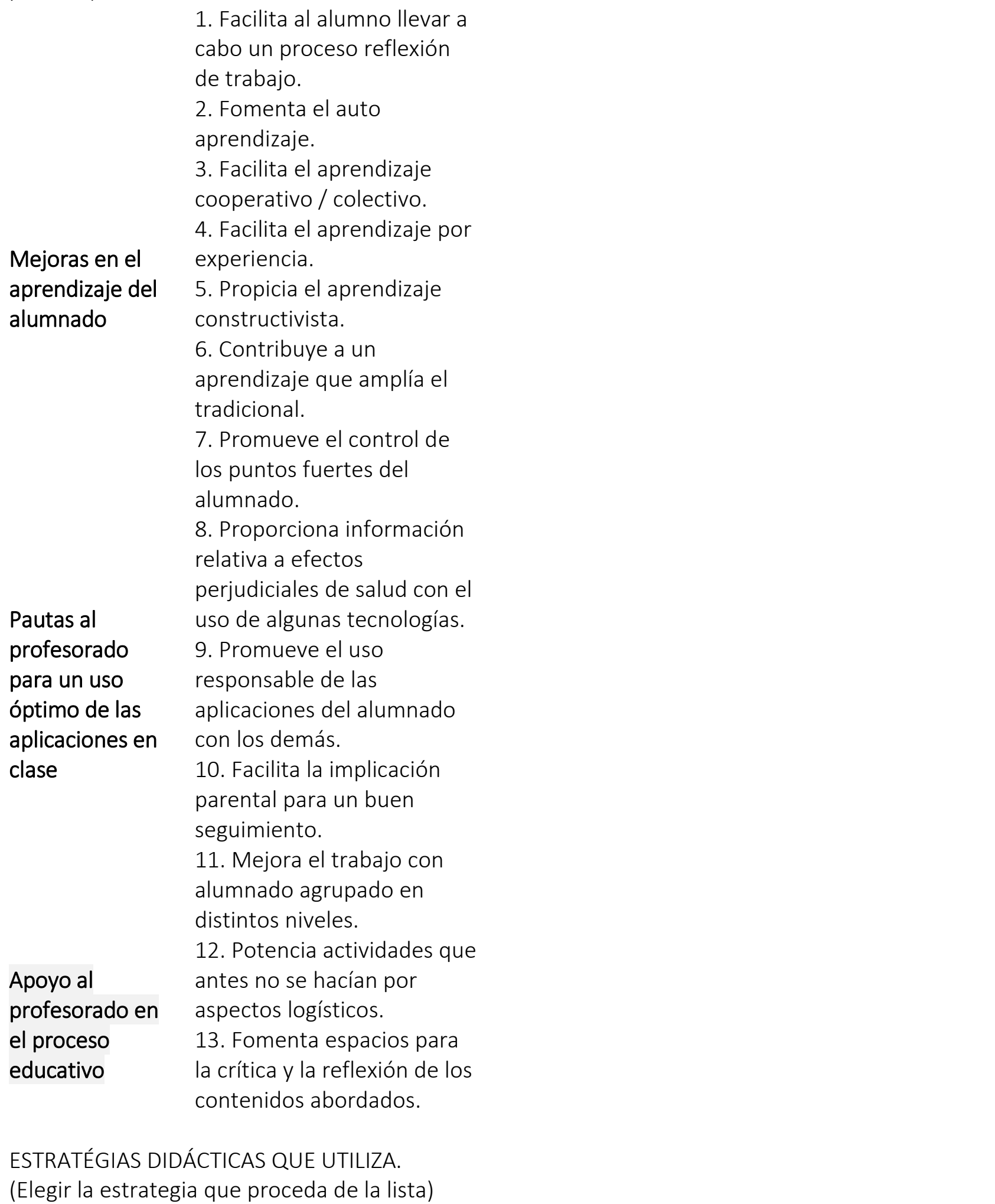


Elija un elemento.

COMPETENCIAS BÁSICAS QUE SE ALCANZAN.

(Marcar con una $\mathrm{X}$ en la casilla que proceda)

COMUNICACIÓN LINGÜÍSTICA

SOCIALES Y CÍVICAS $\square$

Componente lingüístico $\square$

Componente pragmático-discursivo $\square$

SENTIDO DE INICIATIVA Y ESPÍRITU

Componente sociocultural

EMPRENDEDOR

MATEMÁTICA $\square$

CIENCIA Y TECNOLOGÍA $\square$

CONCIENCIA Y EXPRESIONES CULTURALES $\square$

APRENDER A APRENDER $\square$

DIGITAL

OBSERVACIONES generales del uso de la herramientas propuesta

Positivas:

VALORACIÓN GLOBAL
Negativas:

$\begin{array}{lll}\begin{array}{l}\text { Muy Baja Normal Alta } \\ \text { baja }\end{array} & \begin{array}{l}\text { Muy } \\ \text { alta }\end{array}\end{array}$

